\title{
Magnetically heated granular gas in a low-gravity environment
}

\author{
Peidong $\mathrm{Yu}^{1,2, *}$, Matthias Schröter ${ }^{3,4, * *}$, Masato Adachi ${ }^{1, * * *}$, and Matthias Sperl ${ }^{1,2, * * * *}$ \\ ${ }^{1}$ Institut für Materialphysik im Weltraum, Deutsches Zentrum für Luft- und Raumfahrt (DLR), 51170 Cologne, Germany \\ ${ }^{2}$ Institut für Theoretische Physik, Universität zu Köln, 50937 Cologne, Germany \\ ${ }^{3}$ Max-Planck-Institut für Dynamik und Selbstorganisation, 37077, Göttingen, Germany \\ ${ }^{4}$ Hypatia Science Consulting, 37081, Göttingen, Germany
}

\begin{abstract}
Magnetic forces are used to heat up thousands of spherical particles under low-gravity. This long range external excitation, combined with the induced particle-particle interactions, results in a homogeneous spatial distribution of the particles. Comparisons with predictions of kinetic theories can hence be carried out. Haff's cooling law is verified qualitatively, while the measured cooling time scale is quantitatively different from the prediction. The high velocity tail of the velocity distribution during homogeneous cooling state (HCS) is measured, while the expected cluster formation after HCS can not be verified by our experiment.
\end{abstract}

\section{Introduction}

For kinetic theories, one great challenge posed by granular gases is the energy dissipation through particle collisions. The focus on dealing with this dissipative term often leads to simplifying assumptions of other terms that can complicate the dynamical equations [1]. One such important assumption, the spatial homogeneity of the particles, though straightforwardly achievable by simulations $[2,3]$ when the periodic boundary condition is adopted, introduced difficulties to the experiments. To achieve a homogeneous system in three-dimension, the weight of the particles in the first place needs to be countered either by levitating the particles [4] or by bringing the whole experiment into a low-gravity environment $[5,6]$, such as a parabolic flight, the drop tower, or a sounding rocket.

The next step is to provide the particles with kinetic energy to reach a certain temperature level $T \sim\langle v\rangle^{2}$. This heating process, unlike that normally used in simulations, can not be completely randomly applied to the particles. The resulting spatial distribution is hence not perfectly homogeneous. Most of the experiments performed under low-gravity use the vibration of the sample cell boundaries to heat the particles, resulting in a warmer region at the borders and a cooler region in the center, i.e., a tendency of particle clustering in the middle of the cell [5]. In this work, we use magnetic force to heat particles of ferromagnetic material. The benefit of using a magnetic thermostat is that all the particles in the sample cell, close to the borders or not, are subject to this energy input. A more homogeneous system can thus be achieved with a high number of particles.

\footnotetext{
*e-mail: peidong.yu@dlr.de

**e-mail: schroeter@ science-consulting.info

***e-mail: masato.adachi@dlr.de

****e-mail: matthias.sperl@dlr.de

A video is available at https://doi.org/10.48448/3tw5-d751
}

Based on a homogeneously heated granular gas system, our goals are to verify three important predictions of kinetic theories for dissipative gases. Firstly, after the external heating is turned off, the system is expected to maintain its homogeneity but cools down because the energy loss is no longer compensated. The cooling behaviour of this homogeneously cooling state (HCS) is described by:

$$
\langle v(t)\rangle=v_{0}(1+t / \tau)^{\gamma},
$$

where $v_{0}$ is the average particle velocity at the beginning of the cooling $(t=0)$, and $\tau$ is the cooling time scale related with system properties including, especially, the collision dissipation. Haff in 1983, using hydrodynamic methods, first proposed this algebraic decaying behaviour (when $t \gg \tau$ ) [7]. If one considers a simplified model of particle collisions, which uses one constant coefficient of restitution $\epsilon$, regardless of the relative velocity $g$ of the two colliding particles, the power of the algebraic decay is $\gamma=1$. If viscoelastic collision model is used, which renders $\epsilon$ dependent on $g, \gamma$ becomes $5 / 6$.

The second important prediction is regarding the velocity distribution of the particles $P(v)$. While for nondissipative gases, $P(v)$ follows the Boltzmann-Maxwell distribution $P(v) \sim \exp \left(-k v^{2}\right)$, energy dissipation through collisions generally reallocates more particles at the higher end of the velocity distribution [1]. During the steady state of heating, kinetic theories predict this overpopulation of high velocity tail to follow $P(v) \sim \exp \left(-k v^{3 / 2}\right)$ [3, 8]. For HCS, although $\langle v(t)\rangle$ keeps decreasing following Eq. 1, the scaled velocity $c=v / v_{T}$, is predicted to follow a single distribution $P(c) \sim \exp \left(-k^{\prime} c\right)$ [8]. Here, the thermal velocity $v_{T}$ is related with $\langle v\rangle$ by a constant prefactor.

This quasi-steady state of HCS can end with the onset of cluster instability, especially when $\epsilon$ is small, i.e., energy dissipates fast through collisions. Simulation works 
have shown different kinds of fascinating clustering patterns, demonstrating this third prediction of kinetic theories $[2,9]$.

Many experimental works have been dedicated to the verifications of these three predictions. Ground experiments, often performed in two dimensions with constant heating to counter the gravity, have shown for high velocity end: $P(v) \sim \exp \left(-k^{\prime \prime} v^{\alpha}\right)$ with $\alpha$ ranging from 0.8 to 2 [10]. Cluster formations during heating have also been reported by various works $[5,11,12]$. Most part of the predictions, however, require measurement of the cooling, which can only be done with low-gravity or levitation environment. In that regard, Haff's cooling has been qualitatively shown with both methods respectively [4, 13], although quantitative verification of the cooling time scale $\tau$ yields significant difference from theoretical values [13]. As for $P(c)$ of HCS and the cluster instability after it, to our knowledge, there is a lack of experimental verifications.

\section{Experimental setup}

Our experiment uses the annual sounding rocket campaign MAPHEUS (Materials Physics Experiments under Weightlessness) organized by DLR-MP (Institute of Materials Physics in Space, German Aerospace Center) [14] as our low-gravity platform. The sounding rocket provides 375 s of low-gravity time with a gravity level of approximately $10^{-5} \mathrm{~g}$. As shown by Fig. $1 \mathrm{a}$ and $1 \mathrm{~b}$, the magnetic heating is applied by eight electromagnets diagonally arranged around a cubic sample cell with a inner dimension of $\sim 5 \times 5 \times 5 \mathrm{~cm}^{3}$.
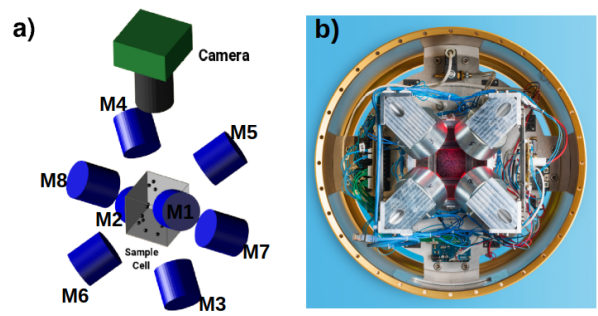

Figure 1. a) Schematics of the setup with eight magnets. b) The top view of the experiment inside the MAPHEUS rocket module. Figures are adapted from Refs. [15, 16]

The PMMA acrylic glass sample cell has an outlet connected to the outside of the rocket to take advantage of the near vacuum environment (air pressure $<0.01 \mathrm{~Pa}$ ) at the high altitude of the rocket $(\sim 100 \mathrm{~km})$. The dilute remnant air inside the cell can thus only apply a weak drag force to the particles, estimated to only reduce $0.01 \%$ of particle velocity per second. Earlier test campaigns showed that particles can adhere to the sample cell boundaries due to static charge. ESLON anti-static coating (by Sekisui Chemical $\mathrm{GmbH}$ ) is therefore applied to the inner surfaces of the rocket sample cell.

A light field Raytrix R5 camera is used to capture the dynamics of the particles under $165 \mathrm{fps}$. Its focusing capability at all depths in the camera view helps us capture more particles than a normal camera can do. The depth information, however, is not precise enough to measure the particle velocities in three dimensions. Therefore, $v$ measured by the camera is indeed the two-dimensional projection of the three-dimensional velocity vector. The relation between the thermal velocity $v_{T}$ in three dimensions and $\langle v\rangle$ measured in two dimensions is thus $v_{T}=2 / \sqrt{\pi} \cdot\langle v\rangle$

More details of the experimental setup is summarized in Ref. [15].

\section{Particles and spatial homogeneity}

2796 spherical MuMetall particles (from Sekels GmbH) with a diameter of $d=1.6 \mathrm{~mm}$ are heated within the inner space of the sample cell. The corresponding packing fraction and number density of the system are $\phi=0.05$ and $n=0.0234 \mathrm{~mm}^{-3}$ respectively.

The coefficient of restitution $\epsilon$ of the MuMetall particles is measured on ground as illustrated in Fig. 2a. One particle is dropped from a finely adjusted position onto another particle fixed below to create an almost vertical headon collision with the collision velocity $g=368 \mathrm{~mm} / \mathrm{s}$. The reduction of the velocity after the collision gives $\epsilon=0.66$.

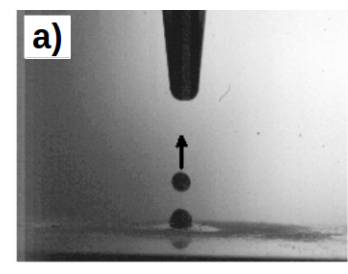

c)
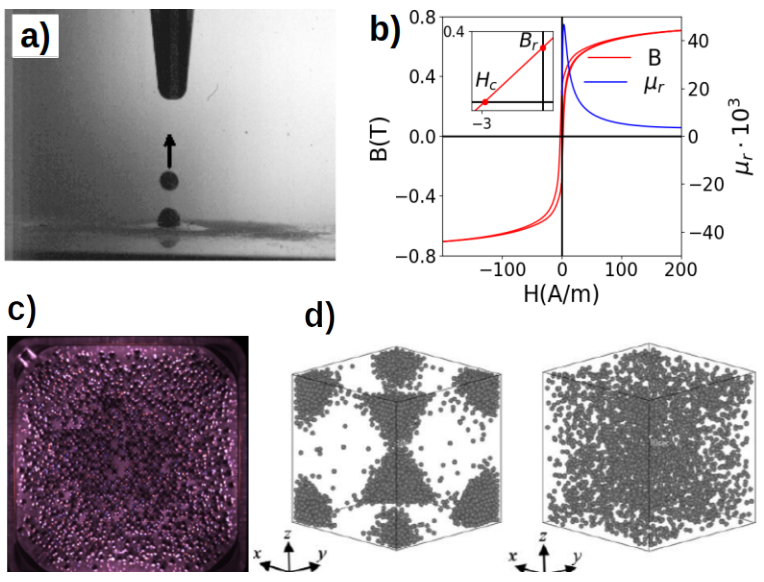

Figure 2. a) A MuMetall particle bouncing back after a collision with another particle fixed below during a lab measurement of $\epsilon$. b) The magnetic hysteresis and $\mu_{r}$ of MuMetall. The inset shows the intersection of $\mathrm{B}-\mathrm{H}$ curve with the negative $\mathrm{x}$-axis: $H_{C}$. c) A snapshot of 2796 heated particles during the rocket experiment. d) Simulation results comparing the case without particle-particle interaction (left) and that with it (right). Figures are adapted from Refs. [15-17].

MuMetall is a ferromagnetic alloy consisting of mainly iron and nickel. The high permeability $\mu_{r}$ of this material (maximum value $\sim 4.5 \times 10^{4}$ ) ensures that the particles are strongly magnetized and quickly heated during the activation period of the heating sequence. On the other hand, MuMetall is also a soft ferromagnet with a very narrow hysteresis loop as shown in Fig. 2b, which leads to a very low coercivity of $H_{C}=2.84 \mathrm{~A} / \mathrm{m}$. During the relaxation period of the heating and the cooling period, the remnant magnetization of our particles $M_{R}=3 H_{C}$ results in a maximum particle-particle attraction force in the order of $10^{-11} \mathrm{~N}$, which is therefore negligible. This soft magnetic property thus removes any significant long-range interactions between the particles and enables a meaningful cooling behaviour comparable with theoretical scenarios. 
With our magnetic heating and the choice of the particle material, an unprecedented large number of particles are successfully heated during the rocket experiment. Fig. $2 \mathrm{c}$ shows one snapshot that demonstrates the homogeneity of particle spatial distribution. See our supplemental video (adapted from Ref. [16]) for more complete heating and cooling behaviours.

A DEM simulation is also performed to reproduce the magnetic heating. Here an interesting finding is the role played by long range particle-particle interactions during the activation period of the heating. Unlike during the cooling, these interactions are indeed strong when the magnets are activated. As shown in Fig. 2d, without considering the particle-particle interaction, the simulation yields particle clusters at the eight corners of the sample cell, while with the interaction calculated, a much better homogeneity is achieved, resembling nicely with the experiment snapshot. Therefore, particle-particle interactions alleviate the strongly directional forces applied by the electromagnets and improve the spatial homogeneity of the heated system. More details of particle properties and simulation results are summarized in Ref. [15, 17].

\section{Results}

Four experiments are performed during the 375 s lowgravity time provided by the sounding rocket. The resulting cooling behaviours and velocity distributions are highly reproducible. Fig. 3a shows the decay of average particle velocity $\langle v\rangle$ of the first experiment. Eq. 1 with $\gamma=1$ is used to fit the cooling. The reproducibility of the experiments is demonstrated when comparing the product of the two fitted parameters $v_{0} \cdot \tau$, which is determined by system properties, from the first experiment with those from the other three. They agree with each other within less than $\pm 3 \%$.

Eq. 2 shows how the product is related with system properties according to kinetic theories only considering a constant $\epsilon$ for the normal component of the relative collision velocity. Here the initial thermal velocity $v_{T 0}$, as mentioned in section 2 , is equal to $2 / \sqrt{\pi} \cdot v_{0}$. $\chi(\phi)$, the contact value of the pair correlation function can be approximated as $\chi(\phi)=(2-\phi) /\left(2(1-\phi)^{3}\right)$ [18]. $a_{2}=16(1-\epsilon)\left(1-2 \epsilon^{2}\right) /\left(81-17 \epsilon+30 \epsilon^{2}(1-\epsilon)\right)$, is the second Sonine expansion coefficient [8]. Using $\epsilon=0.66$ mentioned in section 3 , for the first experiment with fitted $v_{0}=20.6 \mathrm{~mm} / \mathrm{s}$, we obtain a $\tau_{k . t .}=1.34 \mathrm{~s}$, which is much larger than the fitted $\tau=0.507 \mathrm{~s}$. In other words, our system cools significantly faster than the theory predicts.

$$
\tau_{k . t .}=\left[\frac{\sqrt{2 \pi}}{3} \chi(\phi)\left(1+\frac{3}{16} a_{2}\right)\left(1-\epsilon^{2}\right) n d^{2} \cdot v_{T 0}\right]^{-1} .
$$

Fig. $3 \mathrm{~b}$ shows the distribution of scaled velocity $P(c)$ of all four experiments. The deviation from MaxwellBoltzmann distribution for higher $c$ can be clearly seen. A non-linear fitting using $P(c) \sim \exp \left(-k c^{\alpha}\right)$ yields an $\alpha=0.96 \pm 0.19$. This result agrees well with the prediction of kinetic theory.
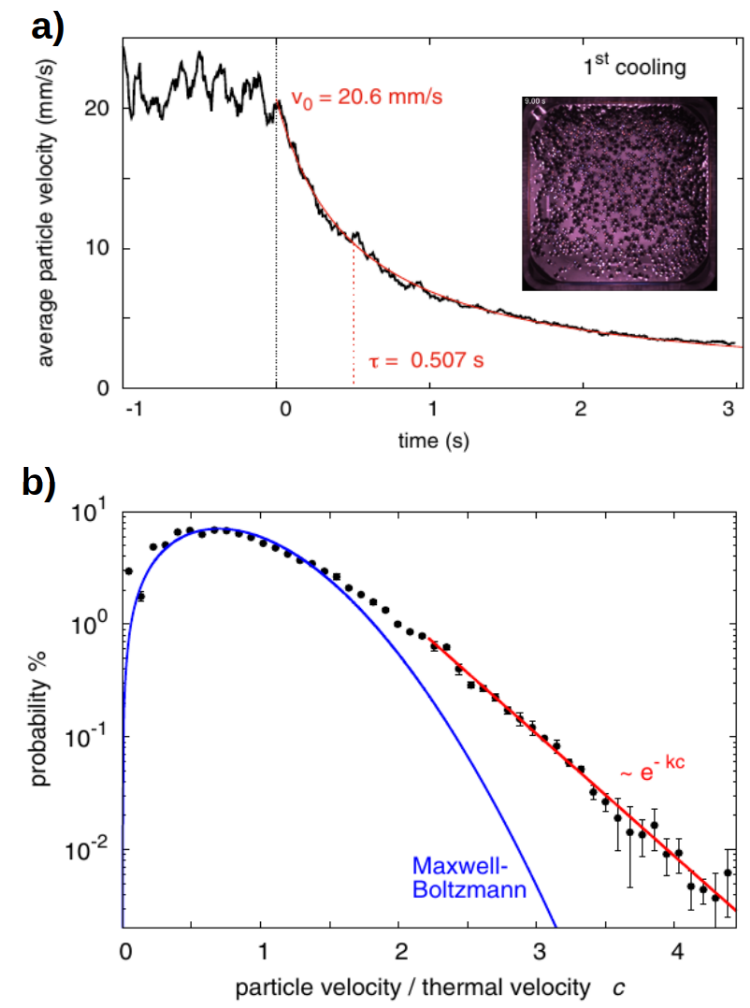

Figure 3. a) The velocity decay of the 1 st experiment fitted by Haff's law. Inset: snapshot taken at 9s after the cooling starts, showing clusters at the top border of the sample cell. b) Velocity distribution of all four experiments during 1.8-3 s after the start of the coolings. Figures are adapted from Ref. [16].

The inset of Fig. 3a shows the formation of clusters at the top border of the sample cell after $4 \mathrm{~s}$ of cooling. We are, however, not convinced that these clusters are formed due to the cluster instability described by the kinetic theory, since a general upward drifting motion of all the particles can be observed by the snapshot and this drifting motion happens in all four experiments. Therefore, it is most likely that the remnant acceleration of the rocket is the underlying reason of the cluster formation. From the motion of the particles we estimate a corresponding acceleration of $3 \times 10^{-5} \mathrm{~g}$, matching the specification of the low-gravity level of the sounding rocket.

More details of the analysis and results are summarized in Ref. [16] and its supplemental materials.

\section{Discussions and conclusions}

Although Haff's law can qualitatively well describe the cooling behaviour of our system, the quantitative difference between $\tau_{k . t .}$ from kinetic theory and $\tau$ from experiment is more than $250 \%$. This difference was similarly observed by another work under low-gravity [13]. One idea to reduce this difference is to use viscoelastic coefficient of restitution $\epsilon=\epsilon(g)$, and the corresponding cooling law of Eq. 1 with $\gamma=5 / 6$ to refit the data. In this case, $\epsilon=0.66$ measured from ground calibration is associated with $g=368 \mathrm{~mm} / \mathrm{s}$. Using kinetic theory for viscoelastic collisions [1], we obtain a cooling time scale $\tau_{v . s .}=0.982$ 
$\mathrm{s}$, while data refitting yields a new $\tau=0.344 \mathrm{~s}$ for the first experiment. The difference between these two values remains larger than $250 \%$.

An obviously missing term is the energy dissipation of the tangential component of particle collisions. Theories taking tangential dissipation into account are readily available $[19,20]$ but involve the rotational motion of the particles and the coupling between rotation and translation. Our current experimental devices are thus not sufficient to measure this additional degree of freedom in three dimensions to verify the theories. These investigations motivate us to improve our diagnostic methods in the future. Before such improvement, we can nevertheless look into the potential of using these theories to reduce the difference. For example, Ref. [19] establishes a dissipation model on the tangential direction using a constant tangential coefficient of restitution $\beta_{0}$ ranging between -1 and 1 , and a dynamic frictional coefficient $\mu$ to address both sticking and sliding motion between two colliding particles. Although $\beta_{0}$ for our particles is unknown, we scan through all possible values and calculate the resulting cooling time scale $\tau_{C}$ in contrast to the time scale $\tau_{k . t}$ for dissipation on normal direction only used in our experiment analysis. As shown in Fig. 4, the difference between $\tau$ and $\tau_{k . t .}$ is partially but never completely reduced for all possible $\beta_{0}$ values. Therefore, a more complicated theory may need to be developed to explain the experiment or the energy partition for different degrees of freedoms assumed by theories (manifested as the constant ratio $r$ used in Fig. 4) need to be first verified with improved diagnostics for our experiment.

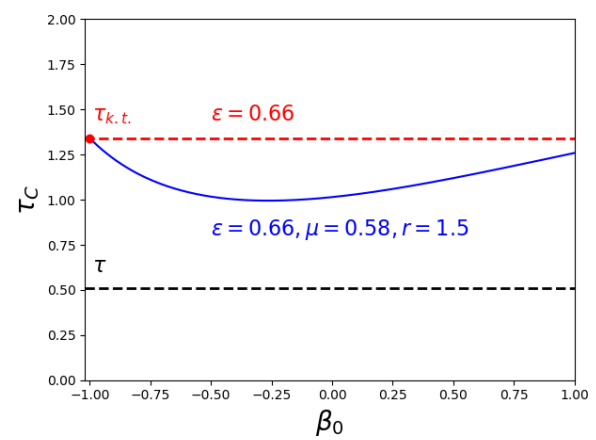

Figure 4. Attempting to reduce the difference between $\tau$ and $\tau_{k . t .}$. using Eq. 18 of Ref. [19]. The coefficient of friction of two sliding MuMetall surfaces $\mu$ is measured in a lab test. $r$, the ratio of rotational and translational energy is assumed to be a constant depending on $\epsilon$ and $\mu$ during the cooling. Using Fig. 6 of Ref. [19], we estimate $r$ to be 1.5.

In summary, the Non-Maxwellian velocity distribution of HCS is soundly verified by our data with an exponentially decaying tail. We conclude that we have not obtained solid evidence of cluster formation after HCS in our system. In addition to the disruption of remnant acceleration, a still lower number of particles compared with simulations (e.g., 40,000 particles in Ref. [2]) may be another underlying reason. Further optimization of our setup to heat even more particles is therefore our future goal.

\section{References}

[1] N.V. Brilliantov, T. Pöschel, Kinetic Theory of Granular Gases (Oxford University Press, 2004)

[2] I. Goldhirsch, M.L. Tan, G. Zanetti, J. Sci. Comput. 8, 1 (1993)

[3] S.E. Esipov, T. Pöschel, J. Stat. Phys. 86, 1385 (1997)

[4] C. Maaß, N. Isert, G. Maret, C.M. Aegerter, Phys. Rev. Lett. 100, 248001 (2008)

[5] E. Falcon, R. Wunenburger, P. Evesque, S. Fauve, C. Chabot, Y. Garrabos, D. Beysens, Phys. Rev. Lett. 83, 440 (1999)

[6] M. Hou, R. Liu, G. Zhai, Z. Sun, K. Lu, Y. Garrabos, P. Evesque, Microgravity Sci. and Tech. 20, 73 (2008)

[7] P.K. Haff, J. Fluid Mech. 134, 401 (1983)

[8] T.P.C. van Noije, M.H. Ernst, Granul. Matter 1, 57 (1998)

[9] P.P. Mitrano, V. Garzó, A.M. Hilger, C.J. Ewasko, C.M. Hrenya, Phys. Rev. E 85, 041303 (2012)

[10] J.S. van Zon, J. Kreft, D.I. Goldman, D. Miracle, J.B. Swift, H.L. Swinney, Phys. Rev. E 70, 040301 (2004)

[11] A. Kudrolli, M. Wolpert, J.P. Gollub, Phys. Rev. Lett. 78, 1383 (1997)

[12] J.S. Olafsen, J.S. Urbach, Phys. Rev. Lett. 81, 4369 (1998)

[13] K. Harth, T. Trittel, S. Wegner, R. Stannarius, Phys. Rev. Lett. 120, 214301 (2018)

[14] M. Siegl, F. Kargl, F. Scheuerpflug, J. Drescher, C. Neumann, M. Balter, M. Kolbe, M. Sperl, P. Yu, A. Meyer, Material Physics Rockets MAPHEUS-3/4: Flights and Developments, in Proceedings of the 21St ESA Symposium on European Rocket and Balloon Programmes and Related Research (2013)

[15] P. Yu, E. Stärk, G. Blochberger, M. Kaplik, M. Offermann, D. Tran, M. Adachi, M. Sperl, Rev. Sci. Instrum. 90, 054501 (2019)

[16] P. Yu, M. Schröter, M. Sperl, Phys. Rev. Lett. 124, 208007 (2020)

[17] M. Adachi, P. Yu, M. Sperl, NPJ Microgravity 19, 1 (2019)

[18] N.F. Carnahan, K.E. Starling, J. Chem. Phys. 51, 635 (1969)

[19] O. Herbst, M. Huthmann, A. Zippelius, Granul. Matter 2, 211 (2000)

[20] A. Bodrova, N. Brilliantov, Granul. Matter 14, 85 (2012) 